\title{
Fditorial
}

\section{Vaccines for prostate cancer : a new era?}

\author{
Julius I Mulia \\ Department of Pharmacy, Medical Faculty, Trisakti University
}

Prostate adenocarcinoma is the most prevalent type of noncutaneous cancer in the Western world, with an estimated 218,890 new cases and 27,050 deaths in the United States in 2007. Currently prostate cancer is detected by measurement of prostate-specific antigen (PSA), a serine protease synthesized by the prostatic epithelium. PSA is an organ-specific and tumor-associated antigen (TAA) but it is not tumor-specific. ${ }^{(1)}$ Partly because of increased cancer screening with PSA, prostatic cancer may now be diagnosed when it is still localized. Localized tumors of the prostate are generally treated with radical prostatectomy, external-beam radiation therapy (EBRT), brachytherapy, or watchful waiting. Unfortunately, up to $30 \%-40 \%$ of patients fail local therapy. The standard treatment of recurrent or metastatic disease is androgen-deprivation therapy (ADT), but this is only a temporary measure as in the majority of cases the cancer ultimately becomes hormone refractory, the condition being termed androgen-independent prostate cancer (AIPC) or hormone refractory prostate cancer (HRPC), which then progresses rapidly. The only available nonpalliative therapy for androgenindependent prostate cancer is docetaxel in combination with prednisone. However, ADT given prior to the onset of clinical symptoms results in rising PSA levels with castrate levels of testosterone, often with a relatively low tumor burden. This systemic treatment earlier in the disease course combined with effective palliative chemotherapy is implicated in the improvement in median survival time of patients with AIPC from an average of about 12 months to about 17-18 months. ${ }^{(2)}$

Clearly there is a need for the development of novel, molecularly targeted therapies of AIPC. It is therefore not surprising that the last decades have seen the development and testing of several types of prostate cancer vaccines as alternative therapeutic modalities. Metastatic prostate cancer is characterized by relatively small lesions within lymph nodes, bone marrow, and other sites within the immune system. Therefore prostate cancer may be more susceptible to vaccine immunotherapy than tumors that are characterized by bulkier or less accessible lesions. ${ }^{(3)}$ Prostate cancer is well suited for an analysis of the efficacy of cancer vaccines because prostate tumors are relatively slow-growing, and early diagnosis of recurrence is frequent. The doubling time of serum PSA acts as a surrogate marker for disease prognosis and outcome. A range of prostate cancer-associated antigens have been identified and characterized, such as secreted proteins PSA and prostatic acid phosphatase (PAP), cell surface proteins prostate-specific membrane antigens (PSMA) and intracellular proteins. Early 
clinical studies have shown that patients will mount immune responses to the prostate cancer-associated antigens post vaccination. ${ }^{(1,4)}$

A number of approaches to prostate cancer immunotherapy have been investigated, such as whole tumor cell, dendritic cell (DC), and poxvirus vaccines. ${ }^{(2)}$ Tumor cell vaccines can be prepared from autologous cells from the patient's own tumor specimen or from cultured allogeneic tumor cells. Autologous cell vaccines contain tumor-specific antigens and TAAs, but their preparation is time-consuming and at times difficult due to tumor necrosis and other conditions decreasing the percentage of tumor cells. On the other hand, allogeneic cell vaccines are relatively easy to prepare and usually consist of one or more tumor cell lines and may also contain several tumor-specific antigens and/or TAAs. The cell lines can also be infected with vectors that express cytokine genes such as granulocyte-macrophage colony-stimulating factor (GM-CSF), or co-stimulatory molecule genes such as B7-1 or B7-2, to enhance immunogenicity of the tumor cells. However, they may not contain essential antigens for a particular patient and there is the potential induction of alloimmunity. ${ }^{(1)}$ A tumor cell vaccine in advanced clinical trial testing is GVAX which consists of two irradiated allogeneic prostate cancer cell lines engineered to secrete GM-CSF. Its mode of action is proposed as the uptake of the ex vivo X-irradiated tumor cells by antigen-presenting cells (APC) and crosspresentation of tumor-associated antigens to T cells in draining lymph nodes. Two phase II clinical studies have been completed in patients with asymptomatic metastatic HRPC, resulting in median survival times of 20 to 35 months. ${ }^{(4)}$

Therapeutic vaccines based on dendritic cells (DCs) carrying tumor antigens have emerged as a promising strategy to initiate an immune response against tumor cells. DC vaccines contain dendritic cells, which are potent APC that have the ability to stimulate T-lymphocyte immune responses in animals and in humans. DCs are capable of activating CD4- and CD8-positive T lymphocytes by antigen presentation on their surface major histocompatibility complex (MHC) class I and class II molecules. DC vaccines are obtained by leukapheresis from peripheral blood mononuclear cells and then cultured in the presence of cytokines before being reinfused into the patient. This process must be conducted for each patient. Apart from the difficult preparation process, DC vaccines also have the disadvantage of high cost. One of the most advanced vaccine approaches is sipuleucel-T, which is not a pure autologous DC vaccine but rather a mixture of white cells. ${ }^{(1)}$ Sipuleucel-T consists of autologous antigen-presenting cells and a fusion protein composed of prostatic acid phosphatase and GM-CSF. In 2006, the results were published on a placebo-controlled, randomized phase III trial comparing sipuleucel-T and placebo in patients with metastatic, asymptomatic, HRPC. Progressionfree survival (the primary endpoint) was not statistically significant, but overall survival between the vaccine and the placebo groups reached statistical significance. ${ }^{(2,4)}$ The vaccine was denied Food and Drug Administration (FDA) approval due to its failure to achieve the primary endpoint, necessitating another study with revised endpoint. For this trial the FDA decision may be published in the coming months of this year.

To stimulate a T-lymphocyte response it is also possible to transfect DCs with tumor messenger RNA (mRNA). This method could be even more potent than loading DCs or APCs with peptides or proteins (as in sipuleucel-T) or transfection with plasmids or transduction with viral vectors. ${ }^{(1,2,5)}$

Poxviruses and fowlpox (modified to be replication-incompetent) have the ability to accept and express multiple transgenes and can thus be engineered to express not only tumor-associated antigens, but also various immunostimulatory molecules. One approach uses recombinant Vaccinia and fowlpox 
vectors containing the transgenes for PSA and three human co-stimulatory molecules (CD80, intracellular adhesion molecule 1, and lymphocyte function-associated antigen 3, designated "TRICOM"). Recent phase I and II trials involving patients with metastatic and locally advanced prostate cancer have shown clinical responses and drops in serum PSA. ${ }^{(2)}$

\section{REFERENCES}

1 Doehn C, Böhmer T, Kausch I, Sommerauer M, Jocham D. Prostate cancer vaccines current status and future potential. Biodrugs 2008;22:71-84.

2 Tarassoff CP, Arlen PM, Gulley JL. Therapeutic vaccines for prostate cancer. Oncologist 2006;11:451-62.

3 Durso RJ, Andjelic S, Gardner JP, Margitich DJ, Donovan GP, Arrigale RR, et al. A Novel alphavirus vaccine encoding prostate-specific membrane antigen elicits potent cellular and humoral immune responses. Clin Cancer Res 2007;13:3999-4008.

4 Schlom J, Gulley JL, Arlen PM. Role of vaccine therapy in cancer: biology and practice. Curr Oncol 2007;14:238-45.

5 Sousa-Canavez JM, Canavez FC, Leite KRM, Camara-Lopes LH. Therapeutic dendritic cell vaccine preparation using tumor RNA transfection: a promising approach for the treatment of prostate cancer. Genet Vacc Ther 2008;6:1-7. 